\title{
Asymptotic Bounds for Frequency Estimation in the Presence of Multiplicative Noise
}

\author{
Zhi Wang and Saman S. Abeysekera \\ School of Electrical and Electronic Engineering, Nanyang Technological University, Block S1, Nanyang Avenue, Singapore 639798
}

Received 29 January 2006; Revised 27 May 2006; Accepted 13 August 2006

Recommended by Vikram Krishnamurthy

\begin{abstract}
We discuss the asymptotic Cramer-Rao bound (CRB) for frequency estimation in the presence of multiplicative noise. To improve numerical stability, covariance matrix tapering is employed when the covariance matrix of the signal is singular at high SNR. It is shown that the periodogram-based CRB is a special case of frequency domain evaluation of the CRB, employing the covariance matrix tapering technique. Using the proposed technique, large sample frequency domain CRB is evaluated for Jake's model. The dependency of the large sample CRB on the Doppler frequency, signal-to-noise ratio, and data length is investigated in the paper. Finally, an asymptotic closed form CRB for frequency estimation in the presence of multiplicative and additive colored noise is derived. Numerical results show that the asymptotic CRB obtained in frequency domain is accurate, although its evaluation is computationally simple.
\end{abstract}

Copyright @ 2007 Hindawi Publishing Corporation. All rights reserved.

\section{INTRODUCTION}

The problem of frequency estimation from noisy signals is of fundamental importance in a variety of applications. Although the performance of frequency estimation in the presence of additive noise is rather well understood, the same can not be stated for frequency estimation in the presence of multiplicative noise. Recently, frequency estimation in the presence of multiplicative noise has received much attention, especially in fading multipath channels, backscatter radar signal processing, and array processing of spatial distributed signals [1-3]. A preliminary step in the development of estimation algorithms in these environments is to identify the fundamental limits of their performance. The Cramer-Rao lower bound (CRB) is a such fundamental lower bound on the variance of any unbiased estimate [4], and is also known to be asymptotically achievable when the number of observations is large.

Computation of the exact CRB in the presence of multiplicative noise has been discussed in $[3,5]$. However, the exact results are usually presented in matrix form that does not offer much insight into the estimation problem that one is dealing with. Furthermore, it is noticed that under high signal-to-noise ratio (SNR) conditions, the covariance matrix involved in $\mathrm{CRB}$ evaluation tends to be singular which makes the derivation of the exact CRB numerically unstable.
This effect is more prominent when the number of observations is large, and in certain multiplicative noise models (e.g., Jake's model), the effect is quite apparent even at a low number of data samples. Similar singularity problems were also encountered in fading channel simulation, minimum mean square error (MMSE) multiuser detection [6, 7]. Parameter estimation with singular information matrices was also discussed in [8], and commonly the singularity is caused by high-dimensional parameter estimation problems via the use of matrix pseudoinverse.

In this paper, to improve numerical stability, we propose to use covariance matrix tapering (CMT) technique when the covariance matrix of the signal is singular. The CMT technique was previously proposed to modify the array pattern in the application of adaptive beamforming [9]. Here in this paper, we will use CMT to resolve the problem of covariance matrix singularity.

For large sample CRB evaluation and computational simplicity, we also propose a DFT-based frequency domain CRB evaluation in conjunction with the CMT technique. In this approach, the asymptotic CRB has been derived using the periodogram of the data in frequency domain [10]. It is noted that this technique is accurate under the condition that the data record length is much larger than the correlation time of the multiplicative noise. Finally, a closed form expression of the large samples CRB for Jake's model is given and a general 
expression for an asymptotic CRB in the presence of multiplicative noise and colored additive noise is provided. The unified approach of the use of CMT in time domain and frequency domain CRB evaluation and a general closed form expression of the CRB for Jake's model are the novel contributions reported in the paper. The closed form expression of the CRB in frequency domain provides direct insight to the accuracy of frequency estimation in different fading channels. Using the expressions for CRB, in Section 4, we have clearly shown how a channel can be characterized under different fading conditions.

Following is an outline of the paper. Section 2 outlines the general signal model encountered in the communication channels. In Section 3, the CRB in time domain in conjunction with the CMT is proposed to solve the singularity problem when the data length and SNR are large. In Section 4, a detailed discussion on the asymptotic CRB at different fading channels is described. In Section 5, we derive the closed form expressions for the CRB in the presence of multiplicative noise and additive colored noise. Our conclusion followed in Section 6.

\section{SIGNAL MODEL}

Consider a general, discrete-time complex time-varying channel in wireless communications, having a frequency offset between the transmitter and the receiver. The data samples at the receiver can be expressed as

$$
x(n)=\left(\mu e^{j \phi}+a(n)\right) e^{j \omega_{0} n}+v(n), \quad n=0,1, \ldots, N-1,
$$

where $\mu$ and $\phi$ are the amplitude and phase of the signal propagating along the direct path. $a(n)$ is the fading causing multiplicative noise. $\omega_{0}$ is the frequency offset between the transmitter and the receiver. $v(n)$ is the additive noise. $N$ is the number of data samples. The following assumptions are placed on the signal model.

(1) $a(n)$ is a stationary complex Gaussian process which is circular symmetric with zero mean and variance $\sigma_{a}^{2}$. (As noted in [11], circularity is an important property in realistic channels.) Its normalized autocorrelation function is defined as $r_{a}(m)=E\left[a(n+m) a^{*}(n)\right] / \sigma_{a}^{2}$, thus $r_{a}(0)=1$. The Gaussian assumption of $a(n)$ gives rise to the well-known Rayleigh distributed amplitude fading when $\mu=0$, while if $\mu \neq 0$, it is the Rician fading.

(2) $v(n)$ is a sequence of independent, identically distributed complex zero mean Gaussian variable with variance $\sigma_{v}^{2}$, and is independent of $a(n)$. The SNR is defined as SNR $=\left(\mu^{2}+\sigma_{a}^{2}\right) / \sigma_{v}^{2}=\left(\mu^{2} / \sigma_{a}^{2}+1\right) / \sigma_{v}^{2} / \sigma_{a}^{2}=$ $\beta(1+\kappa)$, where $\kappa=\mu^{2} / \sigma_{a}^{2}$ is the Rician factor, and $\beta=$ $\sigma_{a}^{2} / \sigma_{v}^{2}$. Thus it can be seen for Rayleigh fading $(\mu=0$, $\left.\sigma_{a}^{2} \neq 0\right), \operatorname{SNR}=\beta$, while in the classical additive white Gaussian noise environment, SNR $=\mu^{2} / \sigma_{v}^{2}=\kappa \beta$.

(3) $r_{a}(m)$ is assumed to be real valued, this will suffice to ensure a consistent frequency estimation via the algo- rithm proposed in this paper. Otherwise, the phase of $r_{a}(m)$ has to be estimated prior to the frequency estimation. This assumption has been made implicitly by many authors, for example, in ionospheric channels for mobile cellular communications, with the correlation function of the fading process is commonly selected as $J_{0}\left(2 \pi f_{d} \tau\right)$, where $J_{0}(\cdot)$ is the zeroth-order Bessel function of the first kind and $f_{d}$ is the Doppler spread [12].

It is noted that estimation of $\phi$ and $\mu$ can be decoupled from the estimation of $\omega_{0}, \phi$ and $\mu$ can also be estimated once $\omega_{0}$ is estimated [13]. In this paper, we only focus on frequency estimation and its CRB in the presence of multiplicative noise.

\section{BOUNDS FOR FREQUENCY ESTIMATION EVALUATED IN THE TIME DOMAIN}

In this section, we consider the CRB for frequency estimation evaluated in the time domain. The CMT is used to regularize the possible ill conditioning of the covariance matrix in time domain. To begin with, consider the signal model presented in Section 2, note here that in the initial discussion, we assume white noise, which is relaxed in Section 5. Recall that the variance of an unbiased estimate $\hat{\theta}_{i}$ of the parameter $\theta_{i}$ has a lower bound that is given by [4],

$$
\mathrm{E}\left[\left(\hat{\theta}_{i}-\theta_{i}\right)^{2}\right] \geq \mathbf{J}_{i i}^{-1}
$$

where $\mathrm{E}[\cdot]$ denotes ensemble average and $\mathbf{J}_{i i}^{-1}$ is the $i$ th element of the diagonal of the inverse of the Fisher information matrix (FIM) J with its $(i, j)$ th element [4],

$$
\mathbf{J}_{i j}=2 \operatorname{Re}\left(\frac{\partial \mathbf{m}^{H}}{\partial \boldsymbol{\theta}_{i}} \mathbf{R}^{-1} \frac{\partial \mathbf{m}}{\partial \boldsymbol{\theta}_{j}}\right)+\operatorname{tr}\left(\mathbf{R}^{-1} \frac{\partial \mathbf{R}}{\partial \boldsymbol{\theta}_{i}} \mathbf{R}^{-1} \frac{\partial \mathbf{R}}{\partial \boldsymbol{\theta}_{j}}\right)
$$

In the above, $[\cdot]^{H}$ denotes the Hermitian transpose, $\theta$ is the vector consisting of parameters $\theta_{i} \cdot \operatorname{Re}(u)$ is the real part of $u$, tr denotes trace of the matrix, $\mathbf{R}$ and $\mathbf{m}$ are the covariance matrix and mean vector of the received signal. Representing the signal model in (1) in terms of vectors, we obtain

$$
\mathbf{x}=\Lambda\left(\mu e^{j \phi} \mathbf{1}+\mathbf{a}\right)+\mathbf{v}
$$

where $\mathbf{x}=[x[0], x[1], \ldots, x[N-1]]^{T}, \mathbf{v}=[v[0], v[1], \ldots$, $v[N-1]]^{T}, \mathbf{1}=[1,1, \ldots, 1]^{T}, \mathbf{a}=[a[0], a[1], \ldots, a[N-1]]^{T}$, and $[\cdot]^{T}$ denotes vector transpose. $\boldsymbol{\Lambda}=\operatorname{diag}\left[1, e^{j \omega_{0}}, \ldots\right.$, $\left.e^{j \omega_{0}(N-1)}\right]$. Suppose that the covariance matrix of multiplicative noise $a(n)$ is expressed as $\sigma_{a}^{2} \mathbf{R}_{\mathbf{a}}$, then mean vector and covariance matrix of the data vector $\mathbf{x}$ can be expressed as

$$
\begin{gathered}
\mathbf{m}_{\mathbf{x}}=\mu e^{j \phi} \boldsymbol{\Lambda} \mathbf{1}, \\
\mathbf{R}_{\mathbf{x}}=\sigma_{a}^{2} \boldsymbol{\Lambda} \mathbf{R}_{\mathbf{a}} \Lambda^{-1}+\sigma_{v}^{2} \mathbf{I} .
\end{gathered}
$$




\subsection{Exact CRB in the presence of multiplicative noise}

Substituting (5) and (6) into (3), the entries of the FIM can be written as [5],

$$
\begin{gathered}
\mathbf{J}_{\omega_{0}, \omega_{0}}=2 \mu^{2} \mathbf{1}^{T} \mathbf{D R}_{\mathbf{z}}{ }^{-1} \mathbf{D} \mathbf{1}+2 \operatorname{tr}\left(\mathrm{R}_{\mathrm{z}}^{-1} \mathrm{DR}_{\mathrm{z}} \mathrm{D}-\mathrm{D}^{2}\right), \\
\mathbf{J}_{\omega_{0}, \phi}=2 \mu^{2} \operatorname{Re}\left\{\mathbf{1}^{T} \mathbf{D R}_{\mathbf{z}}{ }^{-1} \mathbf{1}\right\} \\
\mathbf{J}_{\phi, \phi}=2 \mu^{2} \mathbf{1}^{T} \mathbf{R}_{\mathbf{z}}{ }^{-1} \mathbf{1}
\end{gathered}
$$

where $\mathbf{R}_{\mathbf{z}}=\sigma_{a}^{2} \mathbf{R}_{\mathbf{a}}+\sigma_{v}^{2} \mathbf{I}, \mathbf{D}$ is a diagonal matrix having the form $\mathbf{D}=\operatorname{diag}[0,1,2, \ldots, N-1]$. The FIM entry for $\mu$ is decoupled from the entries for frequency offset and phase of the multiplicative noise [3], thus we have the CRB for frequency estimation

$$
\operatorname{CRB}\left(\omega_{0}\right)=\frac{\mathbf{J}_{\phi, \phi}}{\mathbf{J}_{\omega_{0}, \omega_{0}} \mathbf{J}_{\phi, \phi}-\mathbf{J}_{\omega_{0}, \phi} \mathbf{J}_{\omega_{0}, \phi}} .
$$

Specifically, if $\mu \neq 0, \sigma_{a}^{2}=0$, the covariance matrix becomes proportional to identity matrix, and the CRB for frequency estimation is simplified to

$$
\mathrm{CRB}\left(\omega_{0}\right)=\frac{2 \sigma_{v}^{2}}{\mu^{2} 1^{H} \mathrm{DD} 1}=\frac{6 \sigma_{v}^{2}}{\mu^{2} N^{3}} .
$$

This is the classical expression for CRB in additive white Gaussian noise [4]. If $\mu=0, \sigma_{a}^{2} \neq 0$, which represents the Rayleigh fading in wireless communications, the CRB for frequency estimation is simplified to

$$
\operatorname{CRB}\left(\omega_{0}\right)=\frac{1}{2 \operatorname{tr}\left(\mathbf{R}_{\mathbf{z}}^{-1} \mathbf{D R}_{\mathbf{z}} \mathbf{D}-\mathbf{D}^{2}\right)} .
$$

Note that the denominator of (10) vanishes for temporally white multiplicative noise which will lead to an infinite CRB, but it is noted here that under this condition, the parameters are unidentifiable.

\subsection{Bounds evaluated in the time domain using $C M T$}

The CRB obtained in (8) and (10) is exact for even a finite length of data $N$. However, for certain fading models, the covariance matrix $\mathbf{R}_{\mathbf{z}}$ can be singular especially when the SNR is high. This singularity is caused by the rapid time variation of the fading resulting in information singular process. Information singular processes are simply those having zero Kolmogorov entropy (or equivalently, those processes which are completely determined by their infinite past) [14]. Hence such processes are deterministic which will cause the eigenvalues of the covariance matrix to be zero according to the prediction theory $[6,14]$. Note that, in wireless communication applications, a commonly used model is Jake's model which has the covariance function $R(\tau)=J_{0}\left(\omega_{d} \tau\right)$, where $J_{0}(\cdot)$ is the zeroth-order Bessel function of the first kind and $\omega_{d}$ is the maximum Doppler frequency. For Jake's model, the covariance matrix tends to be ill conditioned even when the data length is small. The effect is more prominent with the increase in the data length. In the following, we will regularize this information singularity using the CMT. The basic idea of CMT is to multiply the elements of the covariance matrix with different weights, in order to attenuate those elements away from the main diagonal. With the use of CMT, the CRB for frequency estimation in (10) can be re-expressed as

$$
\operatorname{CRB}\left(\omega_{0}\right)=\frac{1}{2 \operatorname{tr}\left(\left(\mathbf{R}_{\mathbf{z}} \circ \mathbf{T}\right)^{-1} \mathbf{D}\left(\mathbf{R}_{\mathbf{z}} \circ \mathbf{T}\right) \mathbf{D}-\mathbf{D}^{2}\right)},
$$

with the symbol $\circ$ representing the Hadamard elementwise product between matrices, and $\mathbf{T}$ denoting a tapering matrix. $\mathrm{T}$ is real, symmetric, and Toeplitz. The following theorems provide useful insight into the underlying stochastic properties of CMT technique. For proofs and more details, see [9].

Theorem 1. If $A, B \in \mathcal{C}^{N \times N}$ are both positive semidefinite matrices, so is $A \circ B$. Moreover, if $A$ is positive definite and $B$ is positive semidefinite with no zero diagonal entries, then $A \circ B$ is positive definite.

Theorem 2. Let $\mathcal{P}^{N}$ denote the space of complex-valued $N \times N$ positive semidefinite covariance matrices. Then, if $A, B \in \mathcal{P}^{N}$ and with additional property $d_{i}=1$, for all $i: i=1, \ldots, N$, where $d_{i}$ denotes the ith diagonal entry of $A$ and $B$, then $\chi(A) \geq$ $\chi(A \circ B)$, where $\chi(A)$ is the eigenvalue spread of $A$.

Suppose we choose $B$ as $T$, with $T$ being positive definite. Then Theorem 2 suggests $(A \circ T)$ is positive definite and that the eigenvalue spread has been reduced due to the tapering. That is, a singular matrix can be regularized via the use of $T$. Though Theorems 1 and 2 provide the fundamental property of the CMT as a regularization technique, however, they do not provide a general guideline to choose an optimal tapering matrix. In [9], the tapering matrix is chosen as

$$
[\mathbf{T}]_{m n}=\frac{\sin (\alpha \pi|m-n|)}{\alpha \pi|m-n|}=\operatorname{sinc}(\alpha(m-n)),
$$

where $[\mathbf{T}]_{m n}$ is the $(m, n)$ th element of matrix $\mathbf{T}$ and $0 \leq \alpha \leq$ 1 . Note for the special case $\alpha=0$, the tapering matrix defined in (12) becomes a matrix with all ones and thus no regularization is obtained, while for the case $\alpha=1$, the tapering matrix becomes the identity matrix, the covariance matrix $\mathbf{R}_{\mathbf{z}}$ will become a diagonal matrix and the CRB becomes CRB obtained via the periodogram [10]. As an example, Figure 1 illustrates the regularization property of the CMT applied to resolve the singularity problem of the covariance matrix. Consider a Rayleigh fading channel with normalized Doppler frequency $f_{d}=0.01$, and the multiplicative noise is described via Jake's model. The SNR varies from $25 \mathrm{~dB}, 60 \mathrm{~dB}$ to $100 \mathrm{~dB}$. It can be seen that when $\alpha$ decreases from $0.003,0.002$ to 0.001 , the CRB gradually meets the exact CRB $(\alpha=0)$. Therefore, it is evident (from the plot of $\mathrm{SNR}=25 \mathrm{~dB})$ that if $\alpha_{1}<\alpha_{2}$, then $\operatorname{CRB}\left(\alpha_{1}\right)<\operatorname{CRB}\left(\alpha_{2}\right)$, 


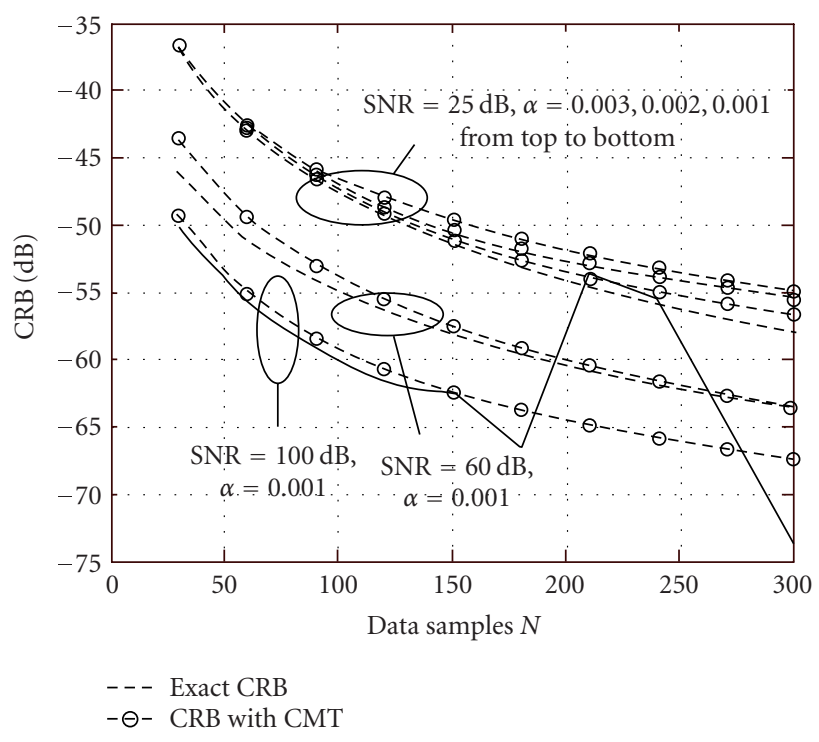

FIgure 1: The exact CRB compared with the CRB when CMT regularization is used for different SNRs, SNR $=25,60,100$ and the normalized Doppler frequency $f_{d}=0.01, \mu=0$.

and $\alpha=0$ provides no regularization to the covariance matrix. Exact value of $\alpha$ selected depends on the required tradeoff between the matrix regularization and the deviation from the CRB. At high $\mathrm{SNR}=100 \mathrm{~dB}$ and large data samples, the singularity of the covariance matrix is prominent. We note from the simulation that the bound obtained using the tapering matrix with $\alpha=0.001$ is very close to the exact CRB while maintaining regular conditions.

\section{BOUNDS FOR FREQUENCY ESTIMATION EVALUATED IN THE FREQUENCY DOMAIN}

The time domain CRB evaluation discussed in the previous section requires matrix inversion. In this section, we elaborate the use of CMT in the evaluation of the CRB in the frequency domain. The major advantage of the frequency domain evaluation is that it avoids matrix inversion and thus it is especially useful when the data lengths are quite large. In particular, we will show that the periodogam-based CRB discussed in [10] is a special case of frequency domain CRB evaluation via the CMT with $\alpha=1$. It is further noted that the periodogam-based CRB can be related to the power spectrum of the signal, thereby providing more insight into characteristics of the estimation problem. We also derive closed form expressions for large samples CRB for Jake's model.

\subsection{Frequency domain CRB with the CMT}

By performing the DFT operation on the data vector $\mathbf{x}$, we obtain

$$
\mathbf{y}=\mathbf{F x}
$$

where $\mathbf{F}$ is the normalized Fourier transform unitary matrix, $\left(\mathbf{F}^{H} \mathbf{F}=\mathbf{I}\right)$, which has the form $\mathbf{F}=\left[\mathbf{e}_{0}, \mathbf{e}_{1}, \ldots, \mathbf{e}_{N-1}\right]$, where

$$
\begin{gathered}
\mathbf{e}_{k}=\frac{1}{\sqrt{N}}\left[1, e^{-j(2 \pi k / N)}, e^{-j(4 \pi k / N)}, \ldots, e^{-j(2 \pi k(N-1) / N)}\right], \\
k=0,1, \ldots, N-1 .
\end{gathered}
$$

$\mathbf{y}$ is the transformed data vector having length $N$. Substituting (4) into (13), we obtain

$$
\mathbf{y}=\sqrt{N} \mathbf{F} \boldsymbol{\Lambda}\left(\mu e^{j \phi} \mathbf{1}+\mathbf{a}\right)+\sqrt{N} \mathbf{F v} .
$$

Consequently, the mean vector $\mathbf{m}_{\mathbf{y}}$ and the covariance matrix $\mathbf{R}_{\mathbf{y}}$ of $\mathbf{y}$ can be expressed as

$$
\begin{gathered}
\mathbf{m}_{\mathbf{y}}=\sqrt{N} \mu e^{j \phi} \mathbf{F} \boldsymbol{\Lambda} \mathbf{1}, \\
\mathbf{R}_{\mathbf{y}}=N \sigma_{a}^{2} \mathbf{F} \boldsymbol{\Lambda} \mathbf{R}_{\mathbf{a}} \boldsymbol{\Lambda}^{H} \mathbf{F}^{H}+\sigma_{v}^{2} N \mathbf{I} .
\end{gathered}
$$

Note that the DFT is a linear reversible operator, and thus it can not be directly used to avoid the singularity associated with $\mathbf{R}_{\mathbf{y}}$. Again, CMT can be employed in the frequency domain to avoid the singularity problem. Equation (17) can be then rewritten as

$$
\mathbf{R}_{\mathbf{t}}=N \mathbf{F} \Lambda \mathbf{R}_{\mathbf{z}} \Lambda^{H} \mathbf{F}^{H} \circ \mathbf{T},
$$

where $\mathbf{T}$ is as given in (12). The $(m, n)$ th element of $\mathbf{R}_{\mathbf{t}}$ can be expressed via matrix expansion

$$
\begin{aligned}
{\left[\mathbf{R}_{\mathbf{t}}\right]_{m n}=} & \frac{e^{j(2 \pi / N)(m-n)}}{N} \sum_{t=0}^{N-1} \sum_{k=0}^{N-1} R_{z}\left(t-k, \omega_{0}\right) \\
& \times e^{-j(2 \pi / N)(t-k)} e^{-j(2 \pi / N)(m k-n t)} \operatorname{sinc}(\alpha(m-n)) .
\end{aligned}
$$

After further manipulation, the above can be written as

$$
\begin{aligned}
{\left[\mathbf{R}_{\mathbf{t}}\right]_{m n}=} & \frac{1}{N} e^{j \pi(m-n)} \sum_{l=0}^{N-1} R_{z}\left(l, \omega_{0}\right) e^{j(\pi(m+n) l / N)} \\
& \times \frac{\sin (\pi(m-n)(N-l) / N)}{\sin (\pi(m-n) / N)} \operatorname{sinc}(\alpha(m-n)) .
\end{aligned}
$$

It can be seen from (19) that using the tapering matrix $\mathbf{T}$, with $\alpha=1, \mathbf{R}_{\mathbf{t}}$ becomes a diagonal matrix

$$
\mathbf{R}_{\mathbf{t}}=\operatorname{diag}\left\{P\left(\omega_{k}, \omega_{0}\right), k=0,1, \ldots, N-1\right\}
$$

with the diagonal element $P\left(\omega_{k}, \omega_{0}\right)$ given by

$$
P\left(\omega_{k}, \omega_{0}\right)=\sum_{l=-(N-1)}^{N-1} w_{B}(l) R_{z}\left(\omega_{0}, l\right) e^{-j \omega_{k} l},
$$

where $\omega_{k}=2 \pi k / N$ and $R_{z}\left(\omega_{0}, l\right)=\sigma_{a}^{2} R_{a}(l) e^{j \omega_{0} l}+\sigma_{v}^{2} \delta(l)$, and $\delta(l)$ is the Kronecker delta function. $w_{B}(l)$ is the Bartlett (triangular) window which is given by

$$
w_{B}(l)= \begin{cases}1-\frac{|l|}{N} & -N+1 \leq l \leq N-1 \\ 0 & \text { elsewhere }\end{cases}
$$


Substituting (16) and (21) into FIM in (3), we obtain the entries of the FIM as

$$
\begin{gathered}
\mathbf{J}_{\omega_{0}, \omega_{0}} \approx \frac{2 \mu^{2} \mathbf{1}^{H} \mathbf{D D} \mathbf{1}}{P\left(\omega_{\gamma}\right)}+\sum_{k=0}^{N-1}\left(\frac{P^{\prime}\left(\omega_{k}, \omega_{0}\right)}{P\left(\omega_{k}, \omega_{0}\right)}\right)^{2}, \\
\mathbf{J}_{\omega_{0}, \phi} \approx \frac{2 \mu^{2} \mathbf{1}^{H} \mathbf{D} \mathbf{1}}{P\left(\omega_{\gamma}\right)}, \\
\mathbf{J}_{\phi, \phi} \approx \frac{2 \mu^{2} \mathbf{1}^{H} \mathbf{1}}{P\left(\omega_{\gamma}\right)},
\end{gathered}
$$

where $\gamma=N \omega_{0} / 2 \pi$ and $P^{\prime}\left(\omega_{k}, \omega_{0}\right)$ is the derivative of $P\left(\omega_{k}, \omega_{0}\right)$ with respect to $\omega_{0}$. The frequency domain evaluation of the CRB obtained via CMT with $\alpha=1$ is in fact the periodogram-based CRB discussed in [10]. The accuracy of the periodogram-based CRB increases with the data samples $N$. As we can see from (20), when $N \rightarrow \infty$, $\left[\mathbf{R}_{\mathbf{t}}\right]_{m n} \rightarrow 0$. Hence, $\mathbf{R}_{\mathbf{t}}$ is asymptotically a diagonal matrix and the periodogram-based CRB asymptotically approaches the exact CRB. Note that by decreasing $\alpha$, the accuracy can be increased but by then it requires matrix inversion, losing the advantage of evaluating CRB in the frequency domain. Also note that when $\alpha=0$, the CRB evaluation in the frequency domain yields the same results as the evaluation in the time domain.

\subsection{Asymptotic expressions for CRB for Jake's model}

Using the periodogram-based CRB evaluation discussed in the previous section, how asymptotic expressions for CRB can be evaluated will be shown here. These expressions are useful because they provide direct insight into how bounds vary with parameters such as number of data points, the Doppler frequency, or the SNR. We consider Jake's model in wireless communication and assume that the length of data samples is much larger than the correlation time of the multiplicative noise. In this case, the power spectrum associated with Jake's model covariance function can be written at discrete frequency points as

$P\left(\omega_{k}\right)= \begin{cases}\frac{2 \sigma_{a}^{2}}{\omega_{d} \sqrt{\left(1-\left(\omega_{k}-\omega_{0}\right) / \omega_{d}\right)^{2}}}+\sigma_{v}^{2} & \left|\omega_{k}-\omega_{0}\right|<\omega_{d}, \\ 0 & \text { elsewhere. }\end{cases}$

Substituting (25) into (24), the FIM entries can be expressed as

$$
\begin{aligned}
\mathbf{J}_{\omega_{0}, \omega_{0}} \approx \frac{2 / 3 \kappa \beta N^{3}}{\left(2 \beta / \omega_{d}\right)+1}+\sum_{k=0}^{N-1}\left(\frac{2 \beta \omega_{k}\left(1-\left(\omega_{k} / \omega_{d}\right)^{2}\right)^{-1}}{2 \beta \omega_{d}^{2}+\omega_{d}^{2}\left(1-\left(\omega_{k} / \omega_{d}\right)^{2}\right)^{1 / 2}}\right)^{2}, \\
\mathbf{J}_{\omega_{0}, \phi} \approx \frac{\kappa \beta N^{2}}{\left(2 \beta / \omega_{d}\right)+1}, \\
\mathbf{J}_{\phi, \phi} \approx \frac{2 \kappa \beta N}{\left(2 \beta / \omega_{d}\right)+1} .
\end{aligned}
$$

Recall that $\kappa=\mu^{2} / \sigma_{a}^{2}$ and $\beta=\sigma_{a}^{2} / \sigma_{v}^{2}$. Therefore, the large sample CRB for frequency estimation is obtained as

$$
\operatorname{CRB}\left(\omega_{0}\right)=\frac{6}{N^{3} \xi+6 \zeta}
$$

where $\xi=\kappa \beta /\left(2 \beta / \omega_{d}+1\right), \zeta=\sum_{k=0}^{N-1}\left(\left(2 \beta \omega_{k}\left(1-\left(\omega_{k} / \omega_{d}\right)^{2}\right)^{-1}\right) /\right.$ $\left.\left(2 \beta \omega_{d}^{2}+\omega_{d}^{2}\left(1-\left(\omega_{k} / \omega_{d}\right)^{2}\right)^{1 / 2}\right)\right)^{2}$. Equation (29) is a closed form expression for CRB at large samples relating to Doppler frequency, data length, and the SNR. It is worth noting that the large sample (asymptotic) CRB can be obtained by approximating the average periodogram by the power spectrum in the continuous form and subsequently using Whittle's formula $[5,15]$. However, the approach used here is direct and more appropriate as the discussed frequency estimate is obtained using discrete data.

It can be seen that, when $\beta=0, \kappa \neq 0,(27)$ provides the $\mathrm{CRB}$ in additive white Gaussian noise. When $\kappa=0$, $\beta \neq 0$, the CRB is entirely determined by (Rayleigh fading) $\zeta$, and the decay rate of the CRB with Doppler spread is in the order of $\omega_{d}^{-4}$. Furthermore, when $\beta \gg 1, \xi$ is simplified to $\xi=\kappa \omega_{d} / 2$, while $\zeta$ can be written as $\zeta=$ $\sum_{k=0}^{N-1} \omega_{k}^{2} / \omega_{d}^{4}\left(1-\left(\omega_{k} / \omega_{d}\right)^{2}\right)$. In this case, the CRB is only determined by $\omega_{d}$ and $\kappa$, and not by SNR, which is known as the floor effect of the fading channels.

Note that under the extremely fast fading condition for Rayleigh fading channel, the multiplicative noise process $a(n)$ can be seen as a case of completely uncorrelated process, that is, $r_{a}(m)=\sigma_{a}^{2} \delta_{m}$, where $\delta_{m}$ is the Kronecker delta function, so that $S_{a}^{\prime}\left(\omega_{k}, \omega_{0}\right)=0, \zeta=0$. Thus CRB becomes infinite, that means in the presence of temporarily white multiplicative noise, no parameters are identifiable. The CRB in the time invariant (slow) fading channel was derived in [3] as

$$
\operatorname{CRB}\left(\omega_{0}\right) \approx \frac{6}{\beta(1+\kappa) N^{3}}
$$

The CRB in the Ricean fast fading channel as $\kappa$ is not too small was approximately derived in [3] as the following:

$$
\begin{aligned}
\operatorname{CRB}\left(\omega_{0}\right) & \approx \frac{2\left(\kappa+1+\rho S_{a}\left(\omega_{0}\right)\right)}{\beta(\kappa+1) \kappa N^{3}} \\
& \approx \frac{2}{\beta(\kappa+1) N^{3}}+\frac{2 S_{a}\left(\omega_{0}\right)}{\kappa N^{3}} .
\end{aligned}
$$

Equation (29) is approximately obtained, and we can see that when $\beta \rightarrow 0$, (29) has the same floor effect of (27). However, (27) also can be used in Rayleigh fading and is a general expression for fading channels.

In Figure 2, we show that for the multiplicative noise with zero mean $(\kappa=0)$ (Rayleigh fading), the CRB increases with the Doppler frequency monotonically. While in Figure 3, $\kappa \neq 0$, (Rician fading) the CRB first increases with Doppler frequency at small values, while beyond that, the CRB decreases with Doppler frequency. The dashed lines are 


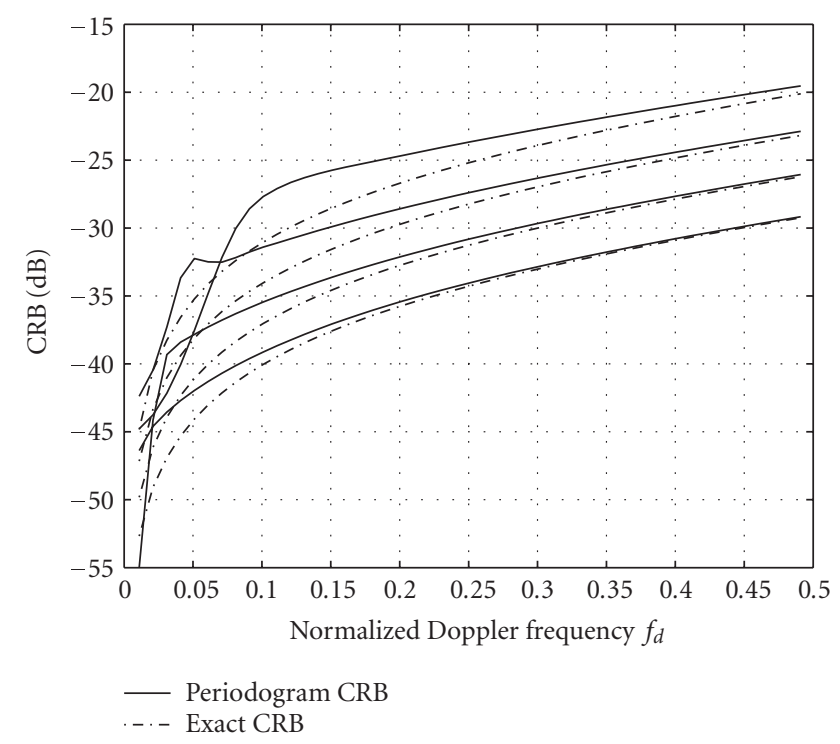

FIGURE 2: The periodogram-based asymptotic CRB and exact CRB with respect to $f_{d}$. The results shown are for different values of $N$. $N=32,64,128,256$, and $\kappa=0 . \beta=10 \mathrm{~dB}$.

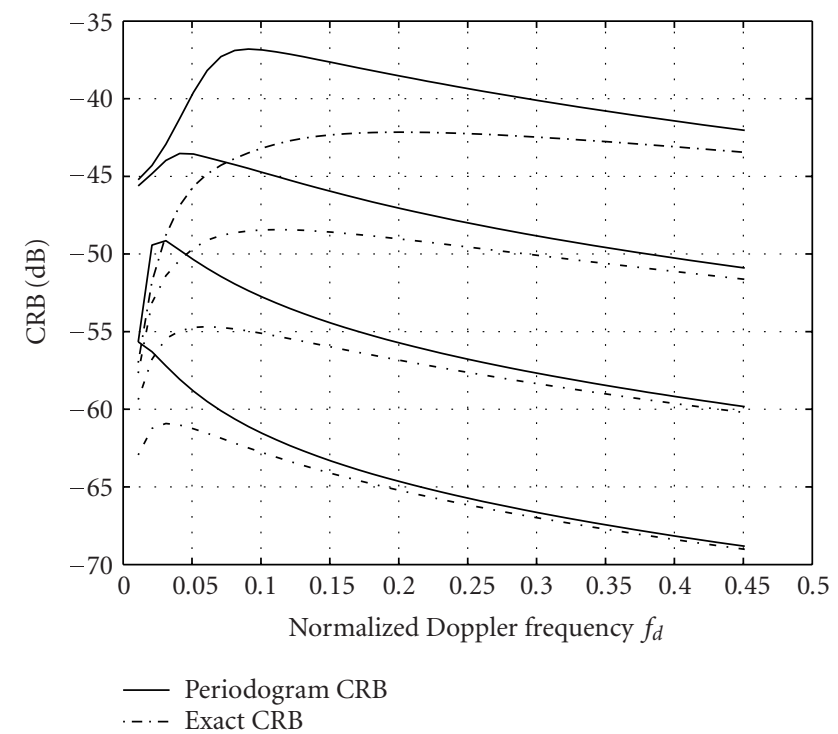

FIgURE 3: The periodogram-based asymptotic CRB and exact CRB with respect to $f_{d}$. The results shown are for different values of $N$. $N=32,64,128,256$, and $\kappa=4 . \beta=10 \mathrm{~dB}$.

the exact CRB and the solid lines represent the large sample asymptotic expressions obtained from (27). It can be seen that the asymptotic CRB meets the exact CRB only at high Doppler frequency and large data samples. This verifies that the periodogram-based CRB expressed in (27) is an asymptotic result mostly suitable for fast fading channels. Figure 4 demonstrates the floor effect of the CRB when $\beta \gg 1$ in the presence of fading channels. The floor effect of Rician channels has been discussed in the literature $[3,5]$. But here, we

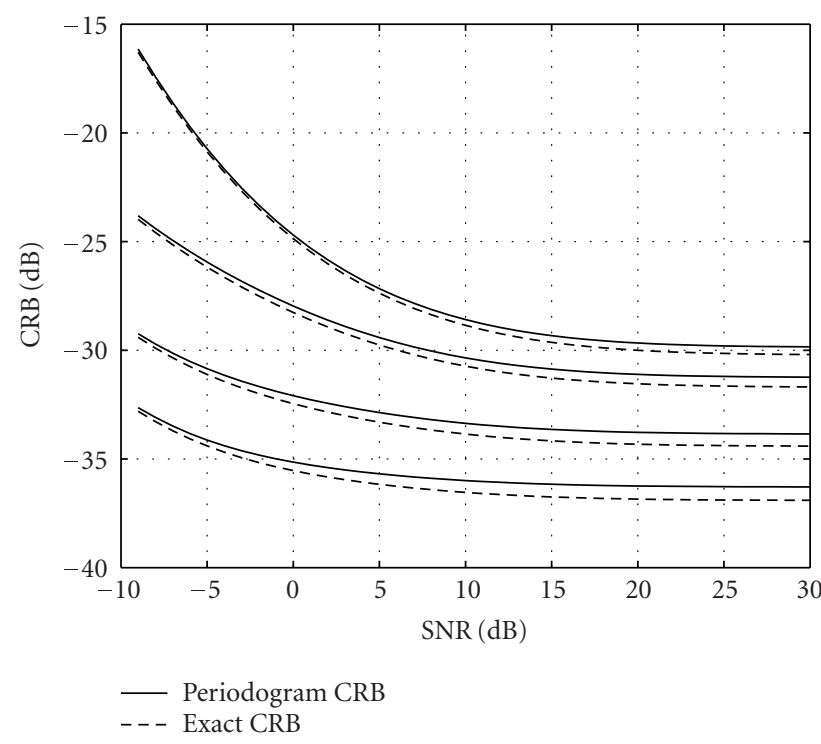

FIGURE 4: The floor effect of the periodogram-based asymptotic CRB and the exact CRB for different values of $\kappa . \kappa=0,0.1,0.2,0.3$ from top to bottom, $N=128, f_{d}=0.3$.

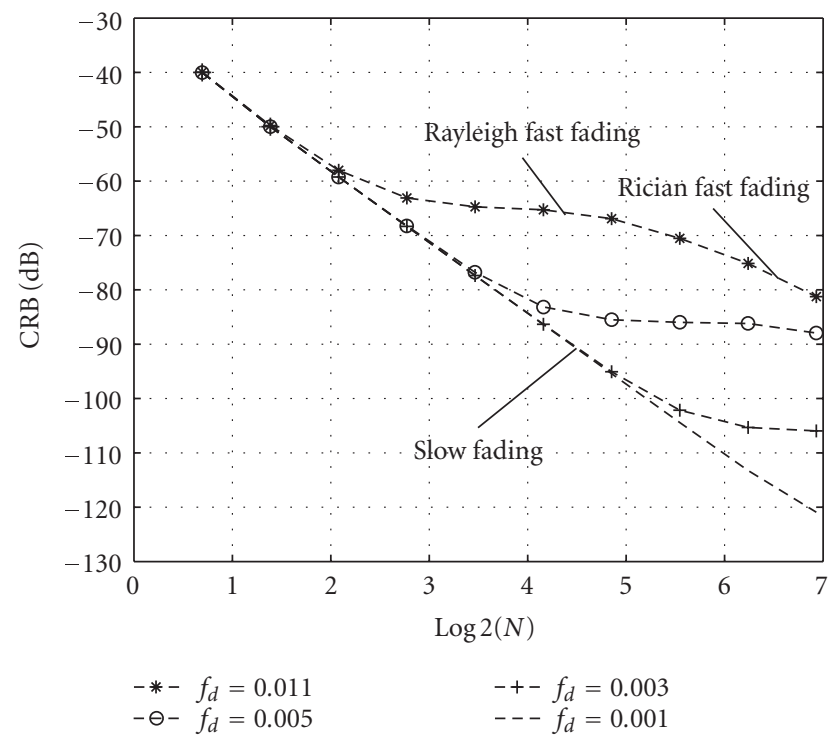

FIGURE 5: The asymptotic CRB with respect to $N$. The results shown are for different values of $f_{d}=0.001,0.003,0.005,0.011$ from top to bottom and $\kappa=100$. $\beta=20 \mathrm{~dB}$.

emphasize that the floor effect is caused by the multiplicative noise, and would be present even in Rayleigh channels.

Figure 5 demonstrates the asymptotic CRB in the presence of multiplicative noise on the effect of data length $N$ for different values of $f_{d}$. It can be seen that when $f_{d}=0.001$, the fading effect is almost negligible, and the channel is similar to an additive white Gaussian noise channel. For large $f_{d}$, for example, $f_{d}=0.011$, the fading effect is determined by the data length $N, \xi$ and $\zeta$ are as shown in (27). In this case, it is 
seen that when $N$ is smaller, the decay rate of CRB is around $N^{-3}$, and the channel behaves as slow fading channel. With the increasing of $N$, the fading relatively becomes fast, and the decay rate of CRB is around $N^{-1}$ which is mainly determined by $\zeta$ in (27), in this situation, the channel can be seen as a Rayleigh fast fading channel. When $N$ is very large, the decay rate of the CRB is around $N^{-3}$ as seen in Figure 5. This is also confirmed by (27) where $\xi$ dominates and the channel behaves as a Rician fast fading channel. Thus knowing $N$, $\xi$, and $\zeta$, using (27), we can determine whether the channel behaves as a slow fading, Rayleigh fast fading, or Rician fast fading channel. That is, the channel can be easily characterized with the use of (27).

\section{CLOSED FORM EXPRESSION FOR CRB IN THE PRESENCE OF MULTIPLICATIVE NOISE AND ADDITIVE COLORED NOISE}

So far in the discussion, we have considered the additive noise as a white process. Through this discussion, the asymptotic CRB expressions for frequency estimation in multiplicative noise and additive white noise have been obtained. In this section, we seek asymptotic CRB expressions in the presence of multiplicative noise and additive colored noise. Without loss of generality, suppose that the colored noise $v(n)$ can be modeled as an order $p$ autoregressive (AR) process, which is expressed via the AR coefficients $a_{k}$ in the following manner:

$$
v(n)=-\sum_{k=1}^{p} a_{k} v(n-k)+e(n) .
$$

Here $e(n)$ is a white Gaussian noise process with variance $\sigma^{2}$. We define that the $\mathrm{SNR}=\left(\mu^{2}+\sigma_{a}^{2}\right) / \sigma^{2}$. Let us first assume that only the colored noise is present (i.e., $\sigma_{a}^{2}=0$ ). Then the inversion of the data covariance matrix $\mathbf{R}_{\mathbf{x}}$ is given by [16],

$$
\mathbf{R}_{\mathbf{x}}{ }^{-1}=\frac{1}{\sigma^{2}}\left(\mathbf{A}_{1} \mathbf{A}_{1}^{H}-\mathbf{A}_{2} \mathbf{A}_{2}^{H}\right)
$$

where $\mathbf{A}_{1}$ and $\mathbf{A}_{2}$ are lower triangular Toeplitz matrices given by

$$
\begin{aligned}
& {\left[\mathbf{A}_{1}\right]_{i j}= \begin{cases}1, & i=j, \\
a_{i-j}, & i>j, \\
0, & i<j,\end{cases} } \\
& {\left[\mathbf{A}_{2}\right]_{i j}= \begin{cases}0, & i<j, \\
a_{N-i+j}^{*}, & i \geq j .\end{cases} }
\end{aligned}
$$

The form in (31) is useful to calculate the exact CRB in the presence of AR colored noise. Substituting (31) into (3), and performing the matrix expansion, the FIM entries in the presence of the AR colored noise are obtained as

$$
\begin{gathered}
\mathbf{J}_{\mu, \mu} \approx \frac{2}{\sigma^{2}}\left|A\left(e^{j \omega_{0}}\right)\right|^{2}(N-3), \\
\mathbf{J}_{\omega_{0}, \omega_{0}} \approx \frac{2 \mu^{2}}{\sigma^{2}}\left(\left|A\left(e^{j \omega_{0}}\right)\right|^{2} \sum_{n=0}^{N-1}(n-2)^{2}+(N-1)^{2}\right), \\
\mathbf{J}_{\phi, \phi} \approx \frac{2 \mu^{2}}{\sigma^{2}}\left|A\left(e^{j \omega_{0}}\right)\right|^{2}(N-1), \\
\mathbf{J}_{\omega_{0}, \phi}=\mathbf{J}_{\phi, \omega_{0}} \approx \frac{2 \mu^{2}}{\sigma^{2}}\left|A\left(e^{j \omega_{0}}\right)\right|^{2} \sum_{n=0}^{N-1} n, \\
\mathbf{J}_{\mu, \omega_{0}}=\mathbf{J}_{\omega_{0}, \mu}=0, \\
\mathbf{J}_{\mu, \phi}=\mathbf{J}_{\phi, \mu}=0
\end{gathered}
$$

where $\left|A\left(e^{j \omega_{0}}\right)\right|^{-2}$ is the normalized spectrum of the AR colored noise and $A\left(e^{j \omega_{0}}\right)=1+\sum_{k=1}^{p} a_{k} e^{-j k \omega_{0}},(k=1,2, \ldots, p)$. Assume that the second term in the right-hand side of equation for $\mathbf{J}_{\omega_{0}, \omega_{0}}$ is small and that the following condition is satisfied:

$$
\left|A\left(e^{j \omega_{0}}\right)\right|^{-2} \ll \frac{N}{3} .
$$

After the matrix inversion, we obtain the asymptotic CRB for frequency estimation in AR colored noise as

$$
\operatorname{CRB}\left(\omega_{0}\right)=\frac{6 \sigma^{2}\left|A\left(e^{j \omega_{0}}\right)\right|^{-2}}{\mu^{2} N\left(N^{2}-1\right)} .
$$

This is in accordance with the asymptotic CRB for short data length as discussed in [16]. Further investigation has revealed that for other colored noise, such as MA colored noise, the results are entirely similar as that of the AR colored noise, provided that the normalized spectrum of the colored noise is much smaller than data length, that is, the condition in (34) is satisfied. Hence we are led to the conjecture that the CRB in colored noise can be obtained by modifying the variance of white noise to accommodate the true AR spectral noise density at the sinusoidal frequency. Following the above, the obvious modification to (27) for the case of colored AR noise is given by replacing $\beta$ by $\beta=\sigma_{a}^{2}\left|A\left(e^{j \omega_{0}}\right)\right|^{2} / \sigma^{2}$, and making $\kappa \beta=\mu^{2}\left|A\left(e^{j \omega_{0}}\right)\right|^{2} / \sigma^{2}$ while keeping $\kappa$ unchanged. The CRB in the time invariant (slow) fading channel in additive colored noise is then given by

$$
\operatorname{CRB}\left(\omega_{0}\right) \approx \frac{6\left|A\left(e^{j \omega_{0}}\right)\right|^{-2}}{\beta(1+\kappa) N^{3}} .
$$

Note that the exact bound can also be obtained from the FIM, where the mean vector is as the same as what appeared in (5). Assuming that the multiplicative noise and additive colored noise are independent, the data covariance matrix can be expressed as

$$
\mathbf{R}_{\mathbf{x}}=\sigma_{a}^{2} \Lambda \mathbf{R}_{\mathrm{a}} \Lambda^{-1}+\sigma_{v}^{2} \mathbf{R}_{\mathbf{v}}
$$

where $\sigma_{v}^{2} \mathbf{R}_{\mathbf{v}}$ is the covariance matrix of the colored noise. Equation (37) can be used to evaluate the exact CRB. Figure 6 compares the exact $\mathrm{CRB}$ and asymptotic CRB expression 


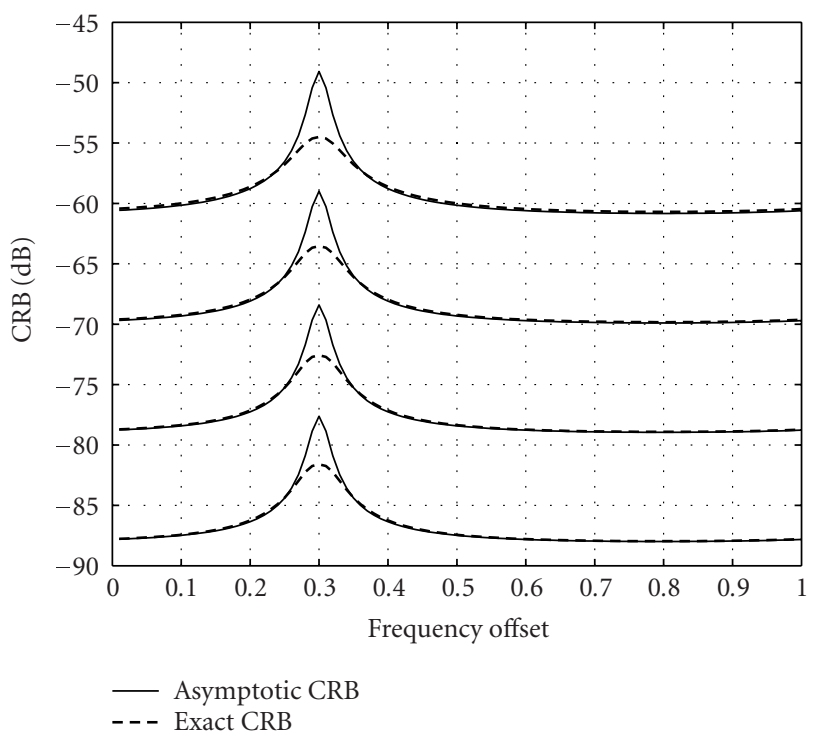

FIgure 6: The asymptotic CRB and exact CRB in the presence of first-order AR colored noise and multiplicative noise for different values of $N, N=32,64,128,256$. SNR $=10 \mathrm{~dB}$. Doppler frequency $f_{d}=0.1 . \kappa=1$.

in multiplicative noise and additive first-order AR noise. In Figure 6, CRB versus frequency offset is shown with the data samples $N$ varying from 32, 64, 128, and 256 from the top plot to the bottom plot. The AR coefficient is set as $a_{1}=$ $0.9 e^{j 0.6 \pi}$. It can be seen that the asymptotic CRB is close to the exact CRB when $N$ is large. Notice that the CRB in the presence of additive colored noise varies with the frequency, although in the presence of additive white noise, it is independent of the frequency. We can also see from Figure 6 that the the CRB peaks at a frequency offset corresponding to the phase of the AR coefficient $a_{1}$, a fact due to the spectral characteristics of the colored noise.

\section{CONCLUSIONS}

Computation of the CRB in the presence of multiplicative noise has been addressed in detail in this paper. It is noted that under high SNR conditions, and in certain multiplicative noise models (e.g., Jake's model), the covariance matrix involved in CRB evaluation tends to be singular which makes the evaluation of the CRB numerically unstable. In this paper, we propose to use CMT technique when the covariance matrix of the signal is singular so as to improve the numerical stability.

We also propose a computationally simple DFT-based frequency domain CRB evaluation method. In this approach, the CRB has been derived using the periodogram of the data. It is noted that this technique is accurate under the condition that the data record length is much larger than the correlation time of the multiplicative noise. Large sample approximations to the CRB for Jake's model is given and a general expression for an asymptotic CRB in the presence of multiplicative noise and colored additive noise is provided. These closed form expressions provide direct insights into the CRB in different fading channels, and help one to obtain proper fading channel characterization.

\section{REFERENCES}

[1] O. Besson, F. Vincent, P. Stoica, and A. B. Gershman, "Approximate maximum likelihood estimators for array processing in multiplicative noise environments," IEEE Transactions on Signal Processing, vol. 48, no. 9, pp. 2506-2518, 2000.

[2] J. Ringelstein, A. B. Gershman, and J. F. Böhme, "Direction finding in random inhomogeneous media in the presence of multiplicative noise," IEEE Signal Processing Letters, vol. 7, no. 10, pp. 269-272, 2000.

[3] F. Gini, M. Luise, and R. Reggiannini, "Cramer-Rao bounds in the parametric estimation of fading radiotransmission channels," IEEE Transactions on Communications, vol. 46, no. 10, pp. 1390-1398, 1998.

[4] S. M. Kay, Fundamentals of Statistical Signal Processing: Estimation Theory, PTR Prentice Hall, Englewood Cliffs, NJ, USA, 1993.

[5] M. Ghogho, A. Swami, and T. S. Durrani, "Frequency estimation in the presence of Doppler spread: performance analysis," IEEE Transactions on Signal Processing, vol. 49, no. 4, pp. 777789, 2001.

[6] K. E. Baddour and N. C. Beaulieu, "Autoregressive models for fading channel simulation," in Proceedings of IEEE Global Telecommunicatins Conference (GLOBECOM '01), vol. 2, pp. 1187-1192, San Antonio, Tex, USA, November 2001.

[7] L. Rugini, P. Banelli, and S. Cacopardi, "Regularized MMSE multiuser detection using covariance matrix tapering," in Proceedings of IEEE International Conference on Communications (ICC'03), vol. 4, pp. 2460-2464, Anchorage, Alaska, USA, May 2003.

[8] P. Stoica and T. L. Marzetta, "Parameter estimation problems with singular information matrices," IEEE Transactions on Signal Processing, vol. 49, no. 1, pp. 87-90, 2001.

[9] J. R. Guerci, "Theory and application of covariance matrix tapers for robust adaptive beamforming," IEEE Transactions on Signal Processing, vol. 47, no. 4, pp. 977-985, 1999.

[10] R. Frehlich, "Cramer-Rao bound for Gaussian random process and applications to radar processing of atmospheric signals," IEEE Transactions on Geoscience and Remote Sensing, vol. 31, no. 6, pp. 1123-1131, 1993.

[11] S. S. Abeysekera, "Performance of pulse-pair method of Doppler estimation," IEEE Transactions on Aerospace and Electronic Systems, vol. 34, no. 2, pp. 520-531, 1998.

[12] J. G. Proakis, Digital Communications, McGraw-Hill, Singapore, 1995.

[13] D. C. Rife and R. R. Boorstyn, "Single-tone parameter estimation from discrete-time observations," IEEE Transactions on Information Theory, vol. 20, no. 5, pp. 591-598, 1974.

[14] B. E. Hajek, "On the strong information singularity of certain stationary processes," IEEE Transactions on Information Theory, vol. 25, no. 5, pp. 605-609, 1979.

[15] A. Zeira and A. Nehorai, "Frequency domain Cramer-Rao bound for Gaussian processes," IEEE Transactions on Acoustics, Speech, and Signal Processing, vol. 38, no. 6, pp. 1063-1066, 1990.

[16] D. N. Swingler, "Approximate bounds on frequency estimates for short cisoids in colored noise," IEEE Transactions on Signal Processing, vol. 46, no. 5, pp. 1456-1458, 1998. 
Zhi Wang received the Master's degree in engineering from Yan Shan University, Qinhuangdao, China, in 2002. He is currently working toward the Ph.D. degree at Nanyang Technological University, Singapore. His research interests are in the areas of signal detection, parameter estimation, and time-frequency domain signal analysis.

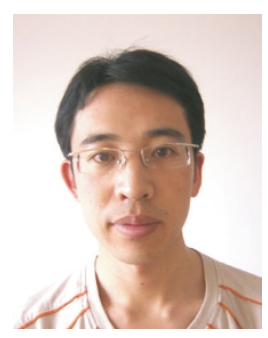

Saman S. Abeysekera received the B.S. degree in engineering (first-class honors) from the University of Peradeniya, Peradeniya, Sri Lanka, in 1978 and the Ph.D. degree in electrical engineering from the University of Queensland, Brisbane, Qld., Australia, in 1989. From 1989 to 1997, he was with the Center for Water Research, University of Western Australia, and Australian Telecommunication Research Institute, Curtin Uni-

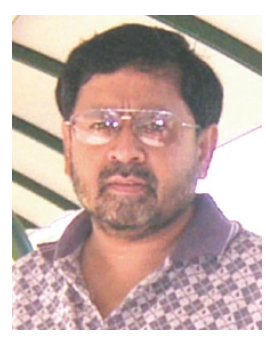
versity of Technology, Perth Australia. He is currently an Associate Professor with the School of Electrical and Electronic Engineering, Nanyang Technological University, Singapore. He is also a Program Director in the Center for Signal Processing. His research interests include frequency estimation, time-frequency domain analysis of audio and electrocardiographic signals, synchronization aspects of SONET/SDH systems, blind signal processing, applications of sigma-delta modulators, and wideband signal processing. 